International Journal of Latest Trends in Engineering and Technology

Vol.(7)Issue(4), pp.165-169

DOI: http://dx.doi.org/10.21172/1.74.021

e-ISSN:2278-621X

\title{
PITCH EXTRACTION AND FUNDAMENTAL FREQUENCY ESTIMATION FROM INDIAN FOLK MUSIC USING TIME DOMAIN AND FREQUENCY DOMAIN TECHNIQUE
}

Shubham Mishra ${ }^{1}$, Rahul Yadav ${ }^{2}$ and Nitin Mukesh ${ }^{3}$

Abstract: In this paper we have applied time domain based and frequency domain based approach for pitch extraction of Indian folk music with pitched instrument in background. After extracting pitch we have also done a comparative study of results. We have analyzed that several melody extraction algorithm were based on pitch extraction or fundamental frequency estimation and now days much advanced algorithm are used for the same. Lot of research work has been done on variety of music but we did not find much articles on Indian Folk music. So in our work we have applied traditional and well established algorithm for pitch extraction and fundamental frequency estimation for Indian folk music.

Keywords-pitch extraction,melody extraction, autocorrelation, cepstrum

\section{INTRODUCTION}

Pitch extraction or fundamental frequency estimation is a prominent research topic of modern time. Pitch is the basic musical component that generally tells about the highness or lowness of a sound. Pitch extraction is widely used for melody extraction for example there are thousands of songs we have heard till date and suddenly if we forget the words of a song and we want to retrieve from our database then in order to search that song we need something called melody concept where we will produce a humming sound and system will come up with corresponding sound result [1]. So it is helpful in searching and interacting with music. There are huge number of application of pitch extraction. Currently there are enormous number of traditional as well as established algorithms for pitch extraction and they are satisfactory to some extent but they did not give the desired results when they are applied on noisy signal or multi pitch signal [4].

Previously lot of techniques has been used for pitch extraction in a previous publication [5]. It is experimentally proved that, a harmonic matching pitch detection algorithm (PDA) is better than

\footnotetext{
${ }^{1}$ Department of Computer Science and Engineering, National Institute of Technology, Agartala, India

${ }^{2}$ Department of Computer Science and Engineering, National Institute of Technology, Agartala, India

${ }^{3}$ Department of Computer Science and Engineering, National Institute of Technology, Agartala, India
} 
Pitch Extraction and Fundamental Frequency Estimation from Indian Folk Music using Time domain and Frequency domain Technique

time domain autocorrelation PDA for vocal pitch detection of music with strong and sparse harmonic spectrum [5]. Researchers have worked with variety of music (Indian classical music, pop music, etc.) In our base paper [1] they have discussed the problem in melody detection of Indian classical music. So in our work what we will be examining is given below:

Q1. Can we directly implement time domain and frequency domain pitch extraction algorithm for pitch extraction from Indian folk music?

Q2. If RQ1 is true then comparative study of results.

\section{LITERATURE REVIEW}

We have applied both autocorrelation method and cepstrum method for pitch extraction of Indian folk music.

\section{- Auto Correlation}

- Initialization takes place with audio signal as input.

- After this waveforms are compared at different time intervals and their similarity at each interval is measured.

- Finally the result is obtained as autocorrelation function which is the Correlation of a waveform with itself.

- This process is done for small time frame and then repeated for whole signal.

$$
\mathrm{R}(\check{\mathrm{r}})=\int \mathrm{x}(\mathrm{t}) * \mathrm{x}(\mathrm{t}+\check{\mathrm{r}}) \mathrm{dt}
$$

\section{- Cepstrum}

Cepstrum is the process that can calculate the fundamental frequency. It uses the technique of calculating the inverse Fourier transformation of logarithmic function of signal. The formulation can be done by using the following formula:

$$
c(t)=\operatorname{IFT}(\log (F T(\text { the signal }))+j 2 \pi m)
$$

The pitch in both the process can be calculated by using the formula

$$
\mathrm{F} 0=1 \mid \check{\mathrm{r} m a x}, \quad \mathrm{r}(\check{\mathrm{r}} \mathrm{max})=\max \quad \mathrm{r} \quad(\check{\mathrm{r}}), \quad \check{\mathrm{r}}>0 \ldots \ldots \text { (iii) }
$$

\section{MOTIVATION}

Most of the pitch extraction method [3] follow the below pattern for Melody extraction.

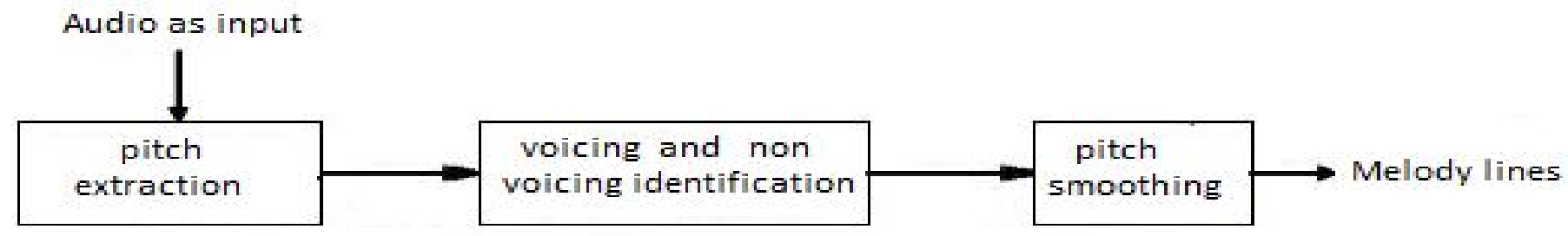

Figure (a) 
Most of the techniques whose aim is to detect vocal melody follows the pattern described in figure(a). So pitch extraction is the fundamental element on which the entire melody detection depends. So after analyzing we came to know that various music have their own characteristic's and depending on that different approach are applied on them for example harmonic matching pitch detection algorithm (PDA) was experimentally found to be superior to the time domain autocorrelation PDA for vocal pitch detection in Indian classical music. So we were motivated to work with Indian folk music since it have its own characteristic's and it was a new point of interest we also wanted to analyze the outcomes when time domain analysis and frequency domain analysis is applied on Indian folk music since we had not seen the results before.

\section{ARCHITECTURE}

System Design

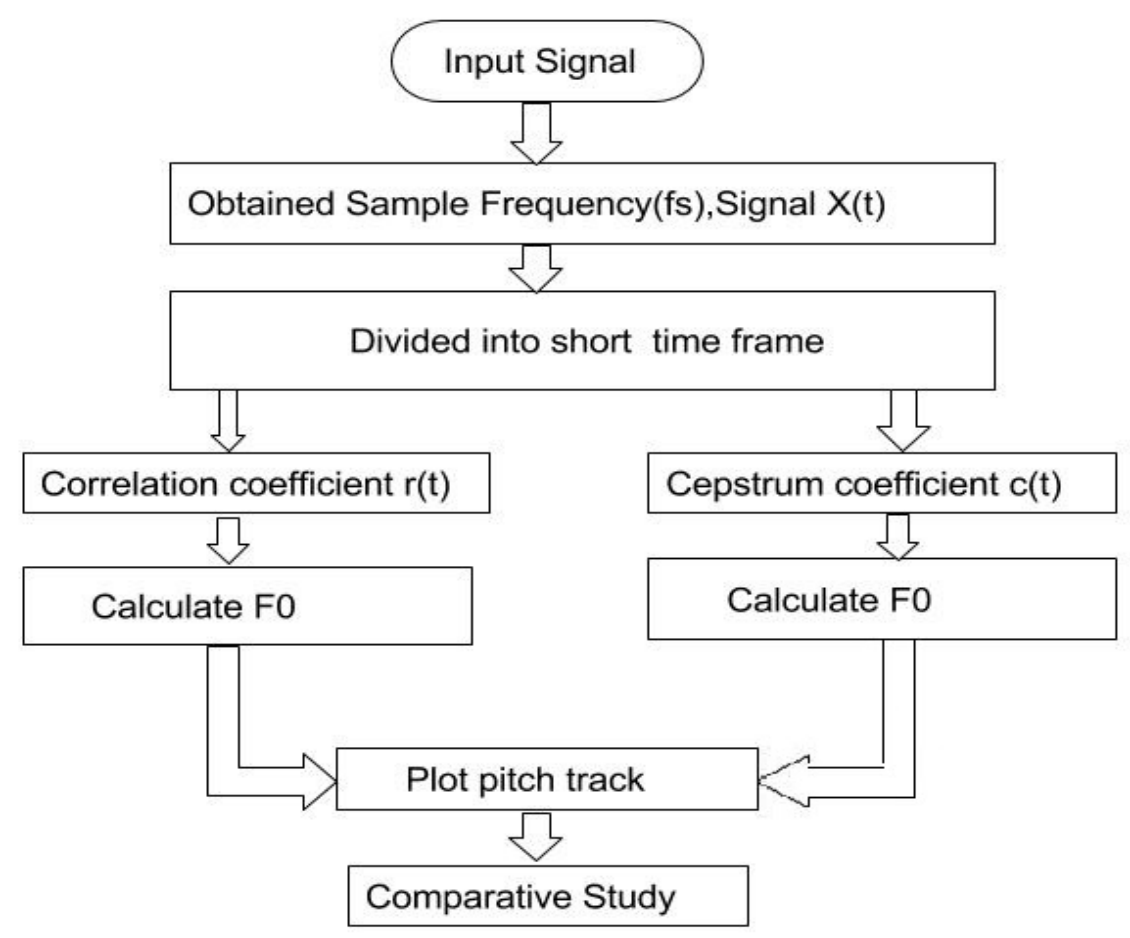

\section{Description}

Figure (b)

Firstly to extract pitch we have taken a Indian folk song as a input and obtained Sampling Frequency (fs) and Vector containing signal $(\mathrm{x}(\mathrm{t}))$. Now we have divided the input in short time frames and will Calculate Correlation Coefficient using Sampling Frequency and signal by calling the correlation function. After this step we will get a vector which contains correlation coefficients. Using correlation coefficient ( $r$ ) and sampling frequency (fs) we will calculate fundamental frequency (f0) for each time frame. Same steps have been followed for cepstrum method pitch extraction. Finally we have plotted tracked pitch on the graph. Using graph and 
Pitch Extraction and Fundamental Frequency Estimation from Indian Folk Music using Time domain and Frequency domain Technique

fundamental frequency we have also served the comparative study purpose.

\section{EXPERIMENTAL SETUP RESULT}
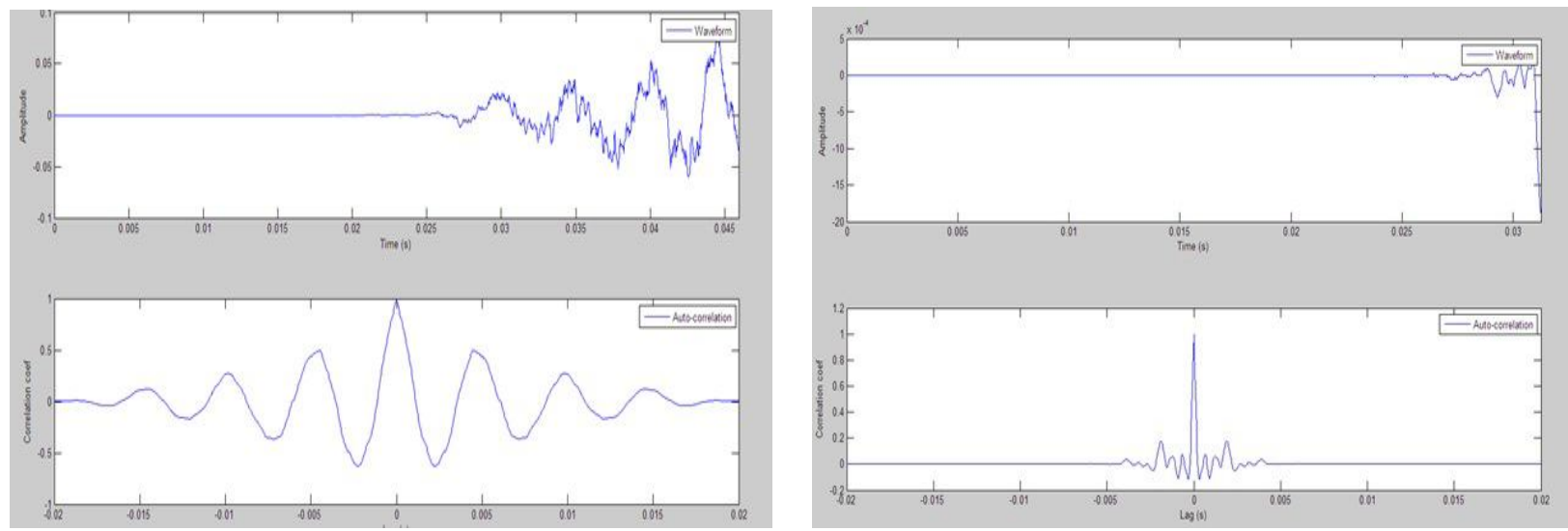

Figure (c) auto correlation coefficient of Nepali song Figure (d) auto correlation coefficient of Bengali song

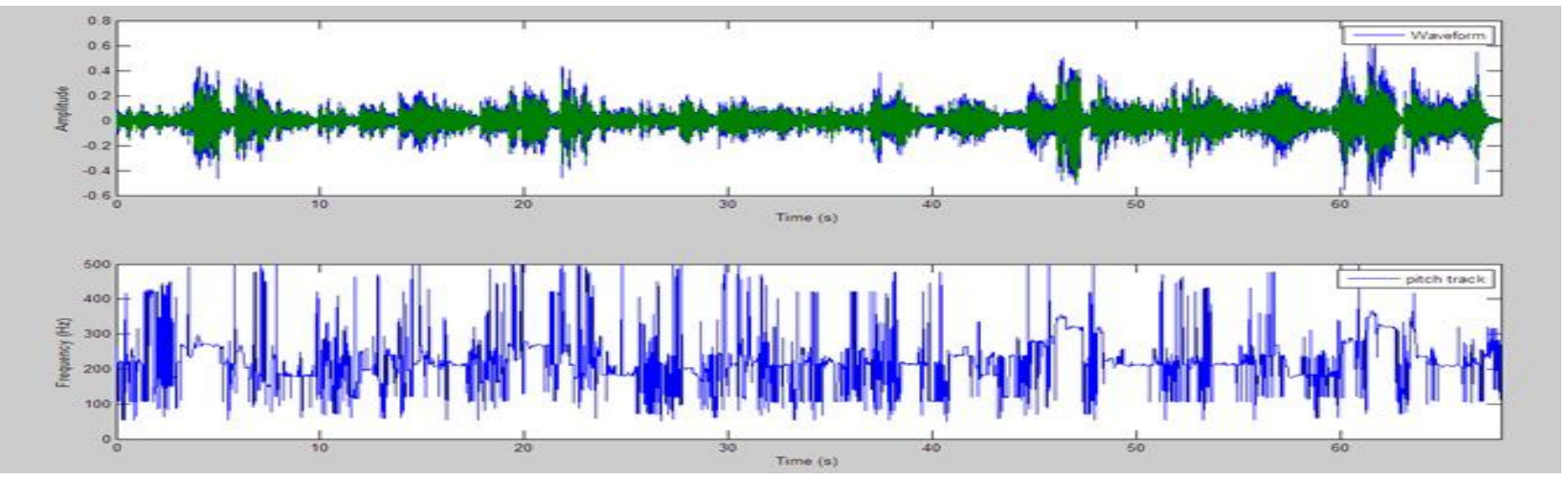

Figure (e) Pitch track of Nepali Folk Song

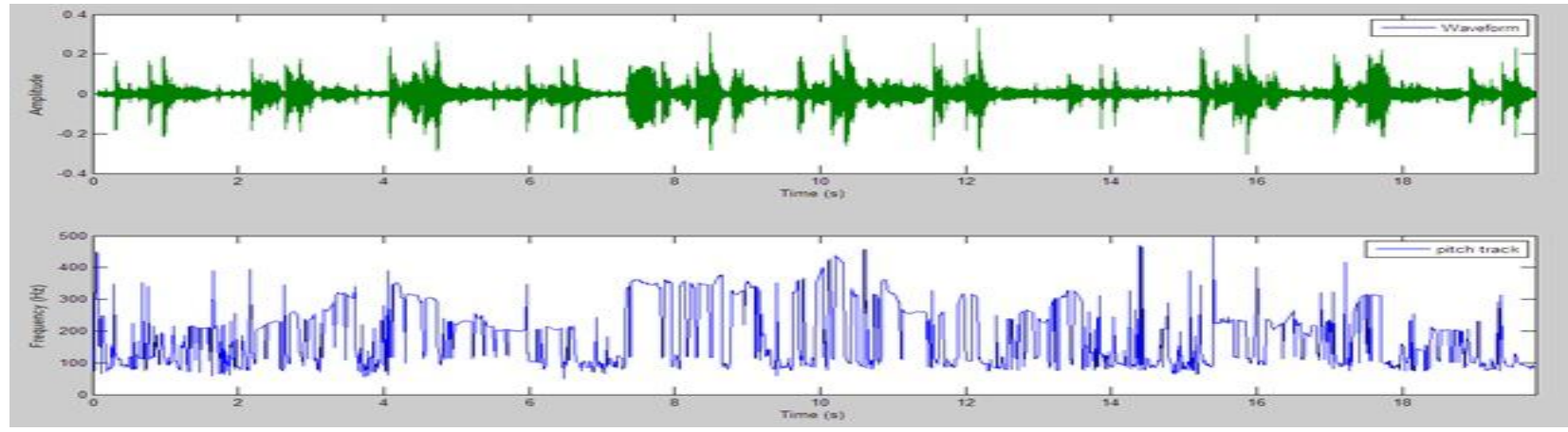

Figure (f) Pitch track of Bengali Folk Song 


\begin{tabular}{|c|c|c|c|c|}
\hline \multirow{2}{*}{ Sample(folk) } & \multicolumn{2}{|c|}{ Auto-Correlation(Time-Domain) } & \multicolumn{2}{c|}{ Cepstrum(Frequency-Domain) } \\
& f0(Hz) & fs(Hz) & f0(Hz) & fs(Hz) \\
\hline Nepali & $217.6871 \mathrm{~Hz}$ & $32000 \mathrm{~Hz}$ & $336.8421 \mathrm{~Hz}$ & $32000 \mathrm{~Hz}$ \\
\hline Bengali & $320 \mathrm{~Hz}$ & $32000 \mathrm{~Hz}$ & $113.8790 \mathrm{~Hz}$ & $32000 \mathrm{~Hz}$ \\
\hline
\end{tabular}

Figure (g) Comparative Study

The figure (e), figure (f) shows the pitch track and figure (c),figure (d) shows correlation coefficient of input Indian folk music respectively using time domain analysis as well as frequency domain analysis. The table in figure $(\mathrm{g})$ shows variation of results when the both approaches (I.e. time domain and frequency domain) applied on the same set of inputs.

\section{CONCLUSION}

The paper has introduced the application of time domain based approach (autocorrelation) and frequency domain based approach (cepstrum) for fundamental frequency estimation and pitch extraction of Indian folk music. Since experimentally we took Indian folk music for the first time. We have also done a comparative study on the results of both method and found variation when two different approaches are applied on same set of samples.

\section{ACKNOWLEDGEMENT}

The authors would like to thanks Sharmistha Majumder, Assistant Professor, Computer Science and Engineering, National Institute of Technology, Agartala, India and National Institute of Technology, Agartala for supporting and guiding us for the completion of this paper.

\section{REFERENCES}

[1] Vishweshwara Rao and Preeti Rao"Vocal Melody Detection In The Presence Of Pitched Accompaniment Using Harmonic Matching Methods" Proc. of the 11th Int. Conference on Digital Audio Effects (DAFx-08), Espoo, Finland, September 1-4, 2008

[2] J. Salamon and E. Gómez, "Melody Extraction from Polyphonic Music Signals using Pitch Contour Characteristics", IEEE Transactions on Audio, Speech and Language Processing, 20(6):1759-1770, Aug. 2012.

[3] G. Poliner, et. al., "Melody Transcription from Music Audio: Approaches and Evaluation," IEEE Trans. on Audio, Speech and Language Processing, vol. 15, no.4, pp. 1247-1256, May 2007.

[4] David Gerhard "Pitch Extraction and Fundamental Frequency: History and Current Techniques" Technical Report TR-CS 2003-06 November, 2003.

[5] A. Bapat, V. Rao and P. Rao, "Melodic contour extraction of Indian classical vocal music," in Proc. Intl. Workshop on Artificial Intelligence and Music (Music-AI ‘07), Hyderabad, India, January 2007. 